\title{
Chapter 8 \\ Atomic-Scale Nanostructures by Advanced Electron Microscopy and Informatics
}

\author{
Teruyasu Mizoguchi, Shin Kiyohara, Yuichi Ikuhara \\ and Naoya Shibata
}

\begin{abstract}
Interfaces dramatically affect the properties of materials because their atomic configurations often differ from the bulk material. A determination of the atomic structure of the interface is, therefore, one of the most significant tasks in materials research. Electron microscopy and theoretical calculations have been effectively used to accomplish this important task. In addition, an informatics approach has recently been combined with theoretical calculations to efficiently determine the atomic structures of interfaces. This chapter introduces the determination of interface structures using an informatics approach (Bayesian optimization and virtual screening) along with advanced electron microscopy. In the informatics approach, calculation acceleration on the order of $10^{6}$ can be achieved. Determination of the interface structure with resolution better than $\sim 45 \mathrm{pm}$ is now possible using advanced electron microscopy. In this way, nanostructures at grain boundaries and heterointerfaces can be qualified. We will introduce these state of the art methods to investigate nanostructures.
\end{abstract}

Keywords Interface - Electron microscopy - Scanning transmission electron microscopy - Bayesian optimization • Virtual screening

\subsection{Atomic Structures of Interfaces}

Interfaces are a kind of lattice defect inside materials and can have significant effects on the overall material properties. For instance, interfaces in polycrystalline materials, i.e., grain boundaries (GB), determine the ion transportation properties and high temperature mechanical properties [1-4]; an atomically controlled interface in a thin film often provides unique properties such as the formation of

T. Mizoguchi · S. Kiyohara · Y. Ikuhara · N. Shibata (四)

The University of Tokyo, Tokyo, Japan

e-mail: shibata@sigma.t.u-tokyo.ac.jp

(C) The Author(s) 2018

I. Tanaka (ed.), Nanoinformatics, https://doi.org/10.1007/978-981-10-7617-6_8 
two-dimensional gases $[5,6]$. The fact that interfaces have different properties from the bulk is a consequence of the fact that they have different atomic structures from the bulk. Thus, for a comprehensive understanding of interface properties, determination of the atomic structure of the interface is crucial.

Since the atomic structure of the interface is strongly dependent on the crystal orientation, lattice planes, and terminations, a systematic study of the interface structure is indispensable for achieving a comprehensive understanding. Thus, the atomic structures of interfaces have already been extensively investigated. Because these characteristic atomic configurations at the interface appear within a very limited area (below $10 \mathrm{~nm}$ ), high spatial resolution observations using transmission electron microscopy (TEM) and theoretical calculations using atomistic simulations have been effectively applied to investigate interfaces.

Similar to TEM, aberration-corrected scanning transmission electron microscopy (STEM) has achieved sub-0.45 $\AA$ spatial resolution [7], and direct atom-by-atom imaging is now routinely possible via annular dark-field (ADF) imaging. In addition, owing to the rapid improvement in detectors, interface chemical analysis using energy-dispersive X-ray spectroscopy (EDS) and electron energy loss spectroscopy (EELS) can also achieve atomic resolution. In short, atomic-resolution STEM has become a very powerful tool for characterizing the atomic structures of interfaces $[8,9]$.

In terms of calculations, extensive calculations are usually necessary to determine even one interface structure because of the geometrical freedom of the interface. Nine degrees of freedom (five macroscopic and four microscopic) are present in an interface. The number of atomic configurations to be considered often reaches $10^{4}$ in even the simplified coincidence site lattice (CSL) grain boundary, namely $\Sigma$ grain boundaries [10, 11]. In a straightforward manner, as schematically illustrated in Fig. 8.1, structure and energy calculations for all candidates must be performed, and leading to optimized configurations and energies of these are obtained $\left(E_{i, j}\right.$ in Fig. 8.1). The most stable configuration with the minimal energy ( $E_{i}$, mim in Fig. 8.1) can then be determined from the density functional theory/ molecular dynamics (DFT/MD) simulation of the interface. Furthermore, the same "brute force" computation is necessary to determine other types of interfaces because the interface structure is dependent on the type of interface $\left(\Sigma \mathrm{GB}_{1}, \Sigma \mathrm{GB}_{2}\right.$, $\ldots \Sigma \mathrm{GB}_{\mathrm{N}}$ in Fig. 8.1).

If the structure and energy of unknown interfaces could be determined more efficiently and accurately, the investigation of interfaces would be dramatically accelerated, which could lead to a deeper understanding of the mechanisms that give rise to interface properties. To more efficiently determine interface structures, a genetic algorithm method and a random structure searching algorithm method have been proposed [13, 14]. However, many trial calculations are still necessary to determine a single grain boundary structure. More recently, much more efficient methods based on machine learning techniques, including virtual screening and Bayesian optimization have been proposed by the present authors [12, 15-17]. Those methods are described below. 


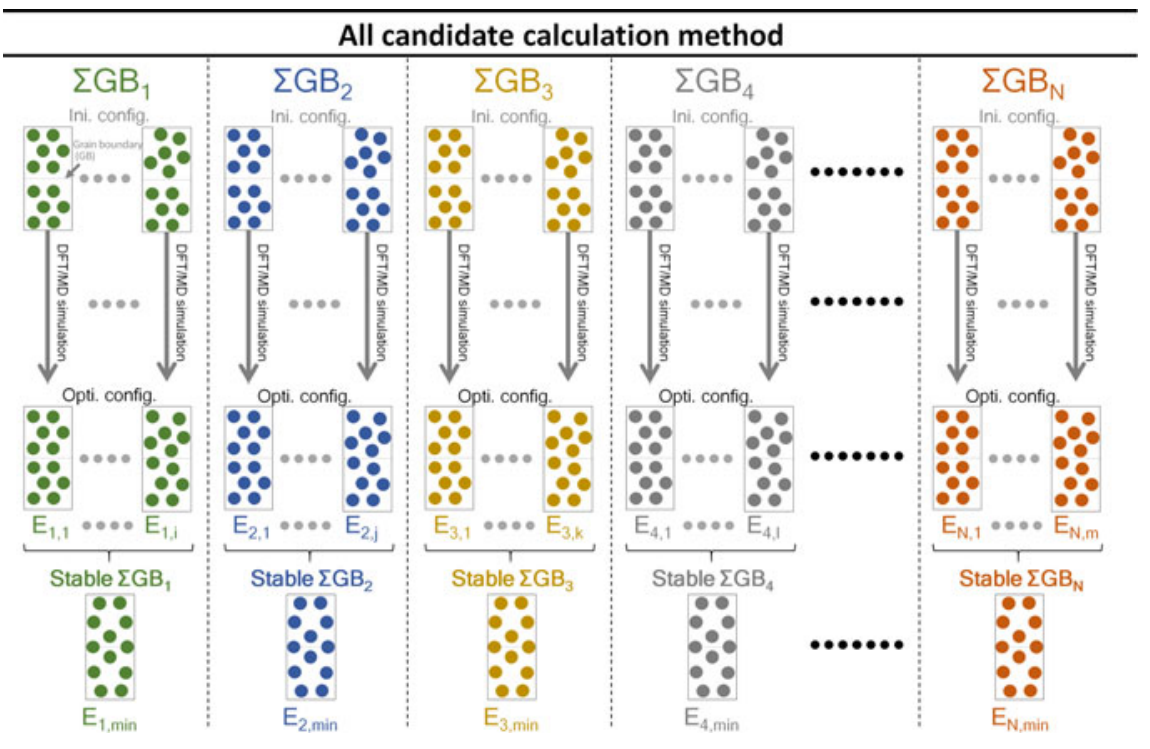

Fig. 8.1 Schematics of all-candidate calculation methods [12]

\subsection{Informatics Approach for Interfaces}

\subsubsection{Virtual Screening}

In this section, a virtual screening method for interface structure determination is described. Virtual screening is an effective method in time-critical problems and was applied to determine the structure and energy of an interface. This virtual screening technique has been used in drug discovery, where a prediction model was constructed using machine learning from a relatively small dataset and a large database consisting of the actual data and data predicted by the prediction model. Then, the candidate drug that is most likely to have the intended effectiveness is selected from the larger constructed database. More recently, this virtual screening method has been applied to discover new molecules for organic electro-luminescence (EL) applications and has succeeded in its discovery aims [18]. We have applied this virtual screening technique to predict the structure and energy of certain interfaces [12].

The idea of our virtual screening method is illustrated in Fig. 8.2. A prediction model (predictor) is constructed via regression analysis of the training data, in this case $\Sigma \mathrm{GB}_{1}$ and $\Sigma \mathrm{GB}_{2}$. Once the predictor is constructed, the grain boundary energies can be predicted from the initial configurations. Then, the candidate configuration that is most likely to give the minimal energy $E_{i, \operatorname{mim}}(i=3,4, \ldots N)$ can be determined. Next, the promising initial configuration is optimized using the 


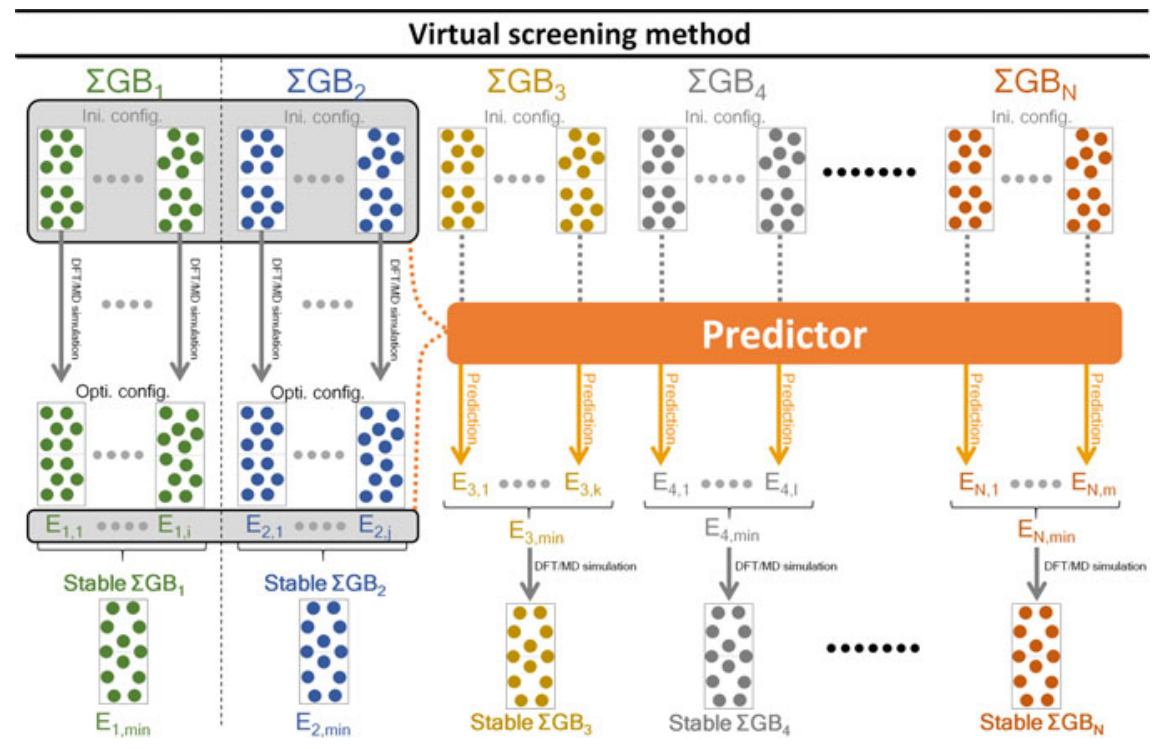

Fig. 8.2 Schematic illustration of virtual screening method for interface structure searching [12]

structure and subsequent energy calculations. Finally, the accurate energy and stable structure are obtained (Stable $\Sigma \mathrm{GB}_{3} \sim \mathrm{N}$ in Fig. 8.2).

Seventeen [001] axis-symmetric tilt CSL grain boundaries of $\mathrm{Cu}$ were considered in this chapter: $\Sigma 5[001] /(210), \Sigma 5[001] /(310), \Sigma 13[001] /(230), \Sigma 17[001] /$ (410), $\quad \Sigma 17[001] /(350), \quad \Sigma 25[001] /(430), \quad \Sigma 25[001] /(710), \quad \Sigma 29[001] /(520), \quad \Sigma 29$ $[001] /(730), \quad \Sigma 37[001] /(610), \quad \Sigma 37[001] /(750), \quad \Sigma 41[001] /(910), \quad \Sigma 41[001] /(540)$, $\Sigma 53[001] /(720), \Sigma 53[001] /(950), \Sigma 61[001] /(1110)$, and $\Sigma 125[001] /\left(\begin{array}{lll}11 & 2 & 0\end{array}\right)$. To obtain stable structures for these grain boundaries, approximately 1,000,000 configurations must be considered. Namely, structure and energy calculations (such as DFT and MD) must be performed 1,000,000 times to determine the structures of these grain boundaries. To construct the predictor, $\Sigma 5[001] /(210), \Sigma 5[001] /(310)$, $\Sigma 17[001] /(350)$, and $\Sigma 17[001] /(410)$ were selected as the training data, corresponding to $\Sigma \mathrm{GB}_{1}$ and $\Sigma \mathrm{GB}_{2}$ in Fig. 8.2. Those grain boundaries were selected as the training data based on the variance of their tilt angles and computational costs for their calculations. Structure and energy calculations for a total of 150,000 configurations, corresponding to approximately $15 \%$ of all possible configurations, were performed. We can confirm that the calculated structures are almost identical to the previously reported structures $[19,20]$, indicating that these training data are suitable for constructing the predictor.

The selection of descriptors for regression analysis is important when predicting the grain boundary energy of non-calculated structures. In this study, geometrical 

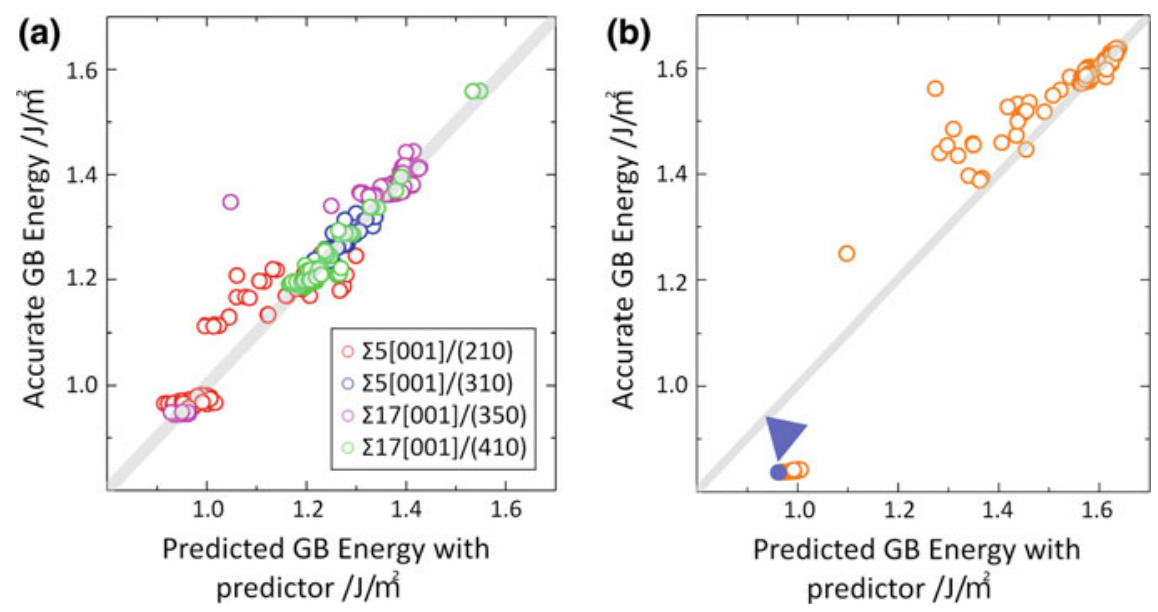

Fig. 8.3 a Results of the regression and $\mathbf{b}$ results for the test data for $\Sigma 13[001] /(230)$ [12]

Table 8.1 List of descriptors

\begin{tabular}{l|l}
\hline \multicolumn{2}{l}{ List of descriptors } \\
\hline $\tan (\theta / 2)$ & Number of longer bond length \\
\hline $\sin (\theta / 2)$ & Average shorter bond length \\
\hline Atomic density around GB & Average longer bond length \\
\hline Average 1st NN (Near Neighbor) bond length & Shortest bond length \\
\hline Average 2nd NN bond length & Number of dangling bond around GB \\
\hline Average 1st NN bond length around GB & Relative translation distance along x direction \\
\hline Average 2nd NN bond length around GB & Relative translation distance along y direction \\
\hline Number of shorter bond length & Relative translation distance along z direction \\
\hline
\end{tabular}

data for the "initial atomic configurations" are used as the descriptors. This choice enables one to predict the grain boundary energy without performing structure and energy calculations. The selected descriptors, such as the minimum and maximum bond lengths are listed in Table 8.1.

In addition to these descriptors, their square, inverse, exponential and exponential inverse values were considered. As a result, 83 descriptors were obtained, which were standardized to align their average and variance to zero and one, respectively.

The nonlinear support vector machine (SVM) method was used for regression analysis. In this study, the most stable structures and metastable structures of $\Sigma 5$ $[001] /(210), \Sigma 5[001] /(310), \Sigma 17[001] /(410)$, and $\Sigma 17[001] /(350)$ were considered for construction of the prediction model. We have selected those grain boundaries as the training data based on the variance of tilt angles and computational costs for their calculations. 
There are two parameters in the SVM, the margin of tolerance and penalty factor. The best parameters were selected from combinations where the margin of tolerance was $0.001,0.01,0.05$ or 0.1 , the penalty factor was $10,100,1000$ or 10000 , and the variance was $10^{-2}, 10^{-3}, 10^{-4}$ or $10^{-5}$, for a total of 64 different patterns. As a result, a margin of tolerance of 0.01 , a penalty factor of 1000 and a variance of $10^{-4}$ were used as SVR parameters.

The results of the regression analysis for the training data are shown in Fig. 8.3a. Most data lie along the grey line, indicating that the predicted energies are equal to the accurate energies and that the regression analysis succeeded in correctly constructing the predictor. To evaluate the accuracy of the constructed predictor, the predictor was applied to $\Sigma 13[001] /(230)$ as a test situation. The results predicted by the predictor are shown in Fig. 8.3b. Most of the predicted grain boundary energies also lie on the grey line, indicating that the constructed predictor is also suitable for the test data. This result implies that the constructed predictor has the potential to predict the energy of the grain boundaries prior to the structure and energy calculations.

Here, we focus on the blue data point marked by the blue arrow in Fig. 8.3b. Based on the constructed predictor, the blue data point was predicted to provide the minimum grain boundary energy. It should be mentioned that the virtual screening method and the calculations of all candidates give the minimum grain boundary energy at the same blue data point. The predicted grain boundary energy is $0.96 \mathrm{~J} / \mathrm{m}^{2}$, which is only $10 \%$ larger than that the minimum grain boundary energy obtained from all-candidate calculations. It is also noteworthy that the predicted rigid body translation state $(\mathrm{X}=5.0 \AA, \mathrm{Y}=1.0 \AA$, and $\mathrm{Z}=0.0 \AA$ ) is identical to the most stable rigid body translation state determined by all-candidate calculations.

We succeeded in screening all possible candidates and selecting the most promising candidate configuration for accurately provide the most stable structure. By performing the structure and energy calculation once for this rigid body translation state, a grain boundary energy and structure identical to those obtained from all-candidate calculations can be obtained. Namely, the stable grain boundary structure and energy can be determined with only a one-time calculation using the present virtual screening method, which is significantly more efficient than previously reported methods.

Since the constructed prediction model (the predictor shown in Fig. 8.2) was established, this predictor was also applied to other GBs. Here, based on the constructed predictor, the structures and energies of 12 other [001]-axis-symmetric tilt CSL grain boundaries, $\Sigma 25[001] /(430), \Sigma 25[001] /(710), \Sigma 29[001] /(520), \Sigma 29$ $[001] /(730), \quad \Sigma 37[001] /(610), \Sigma 37[001] /(750), \quad \Sigma 41[001] /(910), \Sigma 41[001] /(540)$, $\Sigma 53[001] /(720), \Sigma 53[001] /(950), \Sigma 61[001] /(1110)$, and $\Sigma 125[001] /(1120)$, are predicted.

Figure 8.4 shows the results of the predicted grain boundary energies and a comparison with previously reported grain boundary energies [19, 20]. Based on previous studies, the grain boundary energy exhibits a convex profile in relation to the misorientation angle $\theta$. Small cusps are also present, namely energy drops at $16.26^{\circ}, 28.07^{\circ}, 36.87^{\circ}, 53.13^{\circ}$, and $67.38^{\circ}$ corresponding to $\Sigma 25[001] /(710), \Sigma 17$ 


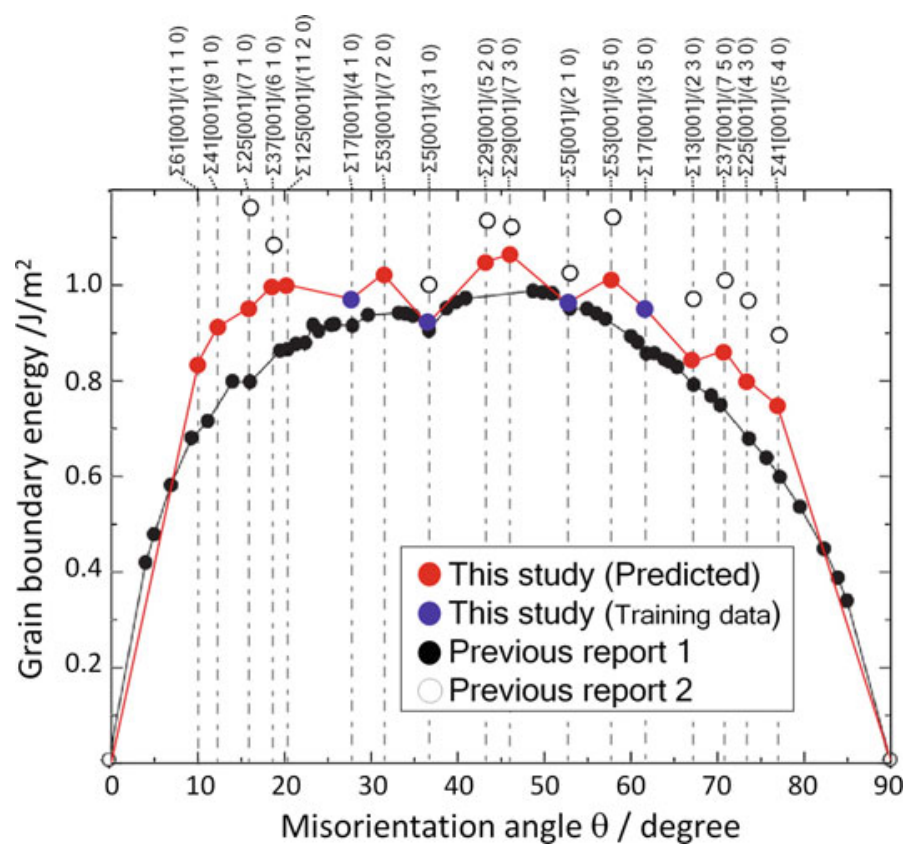

Fig. 8.4 Predicted GB energies using the constructed predictor [15]. Reported values in previous studies are also plotted $[19,20]$

$[001] /(410), \Sigma 5[001] /(310), \Sigma 5[001] /(210)$, and $\Sigma 13[001] /(230)$ respectively. The predicted grain boundary energies of all grain boundaries obtained using the predictor are also plotted in the same figure. Although the absolute value is not identical to the previous studies due to the differences in empirical potential, the overall profile of the grain boundary energy is in good agreement with previous reports. Notably, small cusps at $16.26^{\circ}$ and $67.38^{\circ}$ are also reproduced by the prediction model (other cusps at $28.07^{\circ}, 36.87^{\circ}$, and $53.13^{\circ}$ were used for training). In addition to the GB energy, it was also confirmed that those predicted models fit well to the other calculation and TEM observations. The above results clearly demonstrate that the presented virtual screening method based on machine learning is sufficiently robust and powerful for predicting stable interface structures and energies from initial atomic configurations. The success of this method implies that the initial atomic configuration is correlated to the grain boundary energy, and its correlation is studied by machine learning.

\subsubsection{Bayesian Optimization (Kriging) [15]}

In this section, we demonstrate an alternative and powerful method that can be used to search for stable interface structures with the aid of a geostatistics approach 
called kriging. Kriging is an effective interpolation method based on a Bayesian optimization and Gaussian process governed by prior covariances. This Kriging method has been previously used to predict the optimum access points for geological mining operations. Here, we apply this Kriging technique to determine the stable structures of interfaces.

To demonstrate the performance of the Kriging method, the $\Sigma 5$ [001](210) CSL GB of fcc- $\mathrm{Cu}$ was again selected as the test case. The three-dimensional translations were considered with $0.1 \AA$ steps, resulting in the generation of a total of 17,983 configurations. The data space that must be searched to determine the most stable structure can be visualized as shown in Fig. 8.5a. In the conventional approach, namely all-candidate calculations, one must calculate the interface energies of all configurations and determine the most stable point within this space. In other words, the search space is occupied by the calculated results as shown in Fig. 8.5b.

To accelerate this search process, a Kriging method based on a Gaussian process was applied. The Gaussian process is a nonparametric regression analysis based on Bayesian statistics. This method allows for the prediction of values and uncertainties of a random field at a point. The steps of this Kriging process are as follows:

(1) Several initial configurations (20 configurations here) are randomly selected from the search space shown in Fig. 8.5a.

(2) The structure optimization and energy calculations for the selected configurations are performed, and a joint probability distribution is calculated with the Gaussian process considering the three rigid body translations along each direction as the descriptors. The grain boundary energy, $E_{G B}$, is then estimated.

(3) Based on the joint probability distribution, the possible GB energies are estimated at all points in the search space.

(4) Z-scores, calculated by the following equation, are estimated at all points in the search space.

(a)

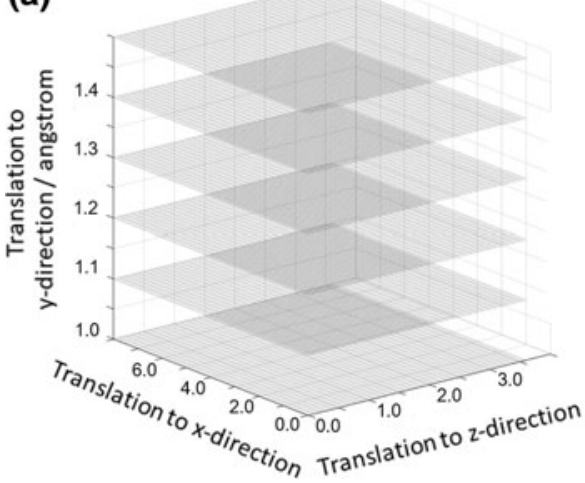

(b)

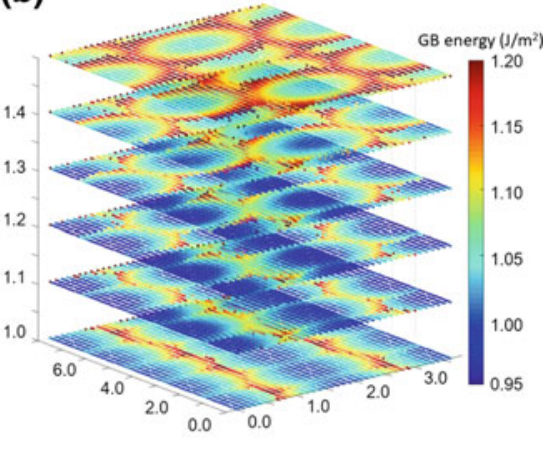

Fig. 8.5 a Data space for searching and $\mathbf{b}$ calculated data space. All data points are calculated [15] 


$$
Z-\text { score }_{i}=\left(G B \text { Energy }_{\text {current } \min }-G B \text { Energy }\left(x_{i}\right)\right) / \sqrt{\sigma\left(x_{i}\right)}
$$

where GB Energy $y_{\text {current min }}$ is the minimum GB energy at this moment, while GB Energy $\left(\boldsymbol{x}_{i}\right)$ and $\sigma\left(\boldsymbol{x}_{i}\right)$ are the mean and standard deviation at the point $\boldsymbol{x}_{\mathrm{i}}$ in the search space respectively.

(5) The point that has the maximum Z-score is selected as the next search point because that point is most likely to have the least GB energy at the moment.

(6) It is confirmed whether or not the acquired GB energies meet the convergence conditions, such as energy difference between the $i$ th calculation and the $i+1$ th calculation.

The cycle of above operations ((2)-(6)) is repeated until the convergence criteria have been satisfied. In the structure optimization and energy calculation for (2), we have performed a static lattice calculation using an empirical potential method with the general utility lattice program (GULP) code [21]. The embedded atom potential method reported by Cleri et al. was employed [22].

First, using the conventional approach, all configurations were calculated and the most stable point was determined from the search space shown in Fig. 8.5b. The obtained stable structure is shown in Fig. 8.6a, where the calculated GB energy was $0.96 \mathrm{~J} / \mathrm{m}^{2}$. As can be seen here, the GB is composed of an array with a six-membered structure unit, in agreement with previously reported structures $[23,24]$. However, 17,983 complete calculations were necessary to reach this stable structure using the conventional all-candidate calculation.

(a)

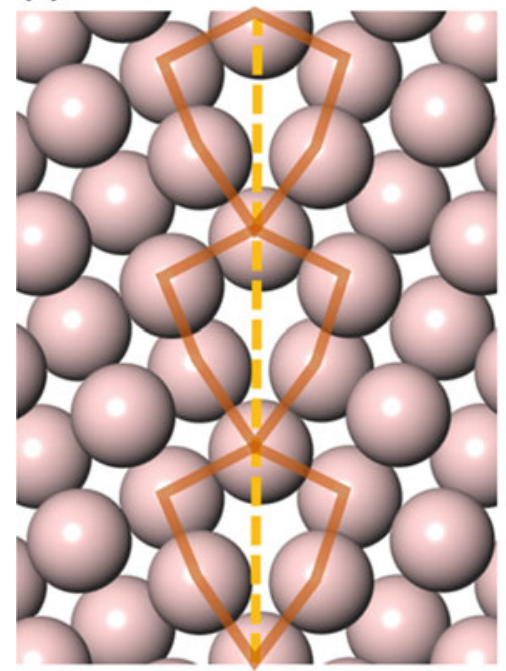

(b)

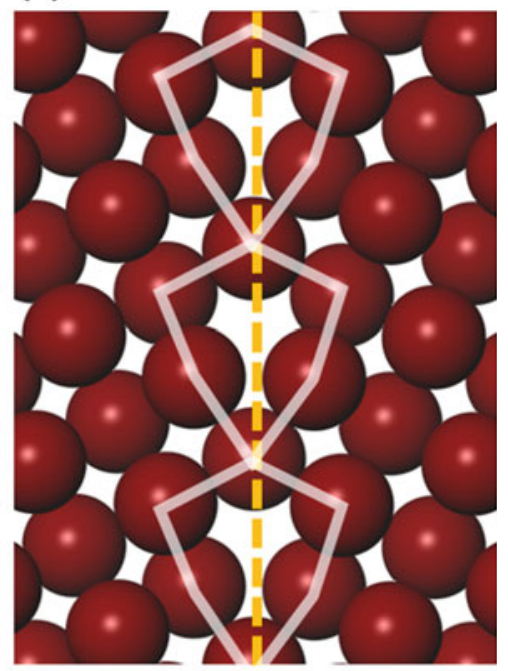

Fig. 8.6 a Calculated structure obtained using the all-candidate calculation and $\mathbf{b}$ using the Kriging method [15] 
On the other hand, in the Kriging approach, the search space was interpolated based on the Gaussian process. We found that this Kriging approach greatly decreased the data necessary for calculations. In this case, the most stable point was determined after only 69 trials (including the initial 20 trials). The most stable structure obtained is shown in Fig. 8.6b; the GB is composed of a six-membered structure unit that is very similar to the stable structure obtained by comprehensive data searching. Furthermore, the calculated GB energy is $0.96 \mathrm{~J} / \mathrm{m}^{2}$, which is identical to that determined by the conventional method, indicating that our present method can accurately determine the most stable structure.

The convergence processes for the all-candidate calculation and the Kriging method are displayed in Fig. 8.7. Although the conventional method requires the calculation of all 17,983 configurations, the present Kriging method requires only 69 calculations (Fig. 8.7a). Figure 8.7b shows the calculation trajectory in the Kriging method. The red numbers show the position in the random sampling, and the pink triangle shows the most stable structure found by the Kriging method. As can be seen in Fig. 8.7b, data space was randomly selected at the beginning of the Kriging method, and it gradually concentrates to neighboring points around the most stable point.

We repeated the Kriging operation 74 times for the same GB and found that the 43 Kriging operations were completed using fewer than 50 time calculations. As a result, the average number of calculations for determining the stable structures is 70. Based on this, the Kriging method has succeeded in accelerating the process of interface structure determination by $\sim 150$ times.

Finally, to confirm the applicability of the Kriging method, a different GB was also examined. The $\Sigma 3[110] /(111) \mathrm{GB}$ of bcc-Fe was selected as a model because its stable structure has also been reported previously [25, 26]. The Kriging method was applied to search for the most stable configuration, just as in the case of the $\Sigma 5$ $[001] /(210)$ copper GB described above. We succeeded in determining the stable structure after 105 calculations, and the calculated structure is shown in Fig. 8.8.

(a)

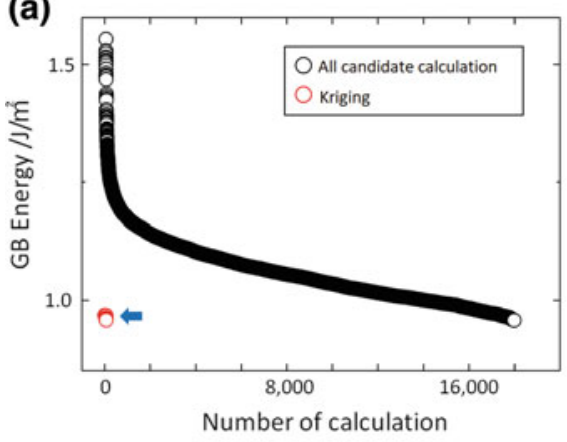

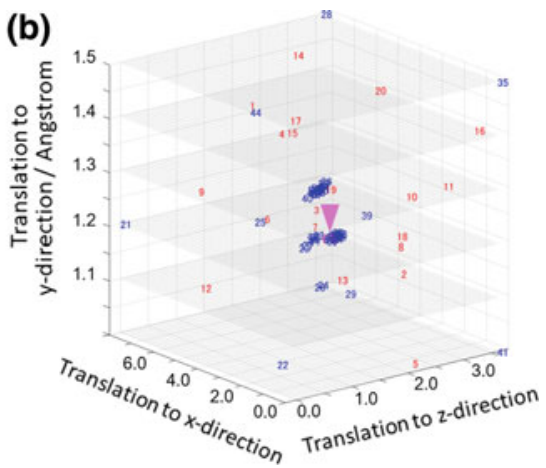

Fig. 8.7 a Number of calculations in both methods. b Calculation trajectory in the Kriging method. Red numbers indicate the position of the initial random sampling and the pink triangle shows the position of the most stable point found by the Kriging method [15] 


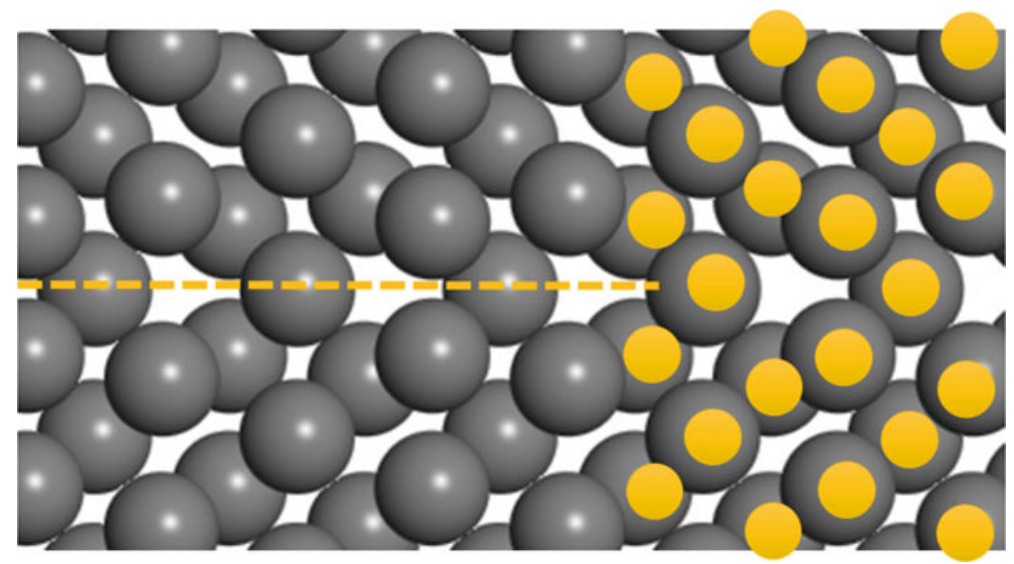

Fig. 8.8 Calculated structure of Fe $\Sigma 3 \mathrm{~GB}$ using the Kriging method [15]. The dashed line represents the position of the GB and the yellow circles show the structure reported previously [25]

The stable structure determined by the Kriging approach agrees well with that of the previous study [25, 26] (yellow circles in Fig. 8.8).

Since 17,466 configurations are present for this $\Sigma 3[110] /(111)$ GB bcc-Fe grain boundary, the Kriging method again achieves two orders of magnitude better efficiency than the conventional method. This clearly indicates that the Kriging method is a very powerful technique to determine the stable interface structure.

\subsubsection{Kriging Method for Oxide Interfaces [16]}

Comparing the virtual screening method and the Kriging method, the efficiency of the virtual screening is superior to that of the Kriging method. However, one has to construct a predictor in order to maintain this great efficiency. The most important advantage of the Kriging method is its wide applicability. No training is needed for the Kriging method, and thus it can be easily applied to other GBs in other materials. To show the wider applicability of the Kriging method, we used it to conduct similar studies on oxide interfaces. In particular, we applied the Kriging approach to grain boundaries of metal oxides including $\mathrm{MgO}, \mathrm{TiO}_{2}$, and $\mathrm{CeO}_{2}$ which commonly exhibit more complex structures than metals.

Four kinds of metal oxide grain boundaries, namely rock-salt-MgO $\Sigma 5[001] /(210)$ and $\Sigma 5[001] /(310)$, rutile- $\mathrm{TiO}_{2} \Sigma 5[001] /(210)$, and fluorite-CeO ${ }_{2}$ $\Sigma 3[110] /(111)$ were selected to test the applicability of the present method. These grain boundaries have different complexities; the number of termination planes for $\mathrm{MgO} \Sigma 5[001] /(210)$ and $\Sigma 5[001] /(310)$ is one (Fig. 8.9a, b), whereas that for $\mathrm{TiO}_{2}$ $\Sigma 5[001] /(210)$ and $\mathrm{CeO}_{2} \Sigma 3[110] /(111)$ is two (Fig. 8.9c, d).

The same Kriging method was applied to these oxides GBs. Two hyper-parameters, pre-distribution and kernel parameter, were set to 0 and 3.0 

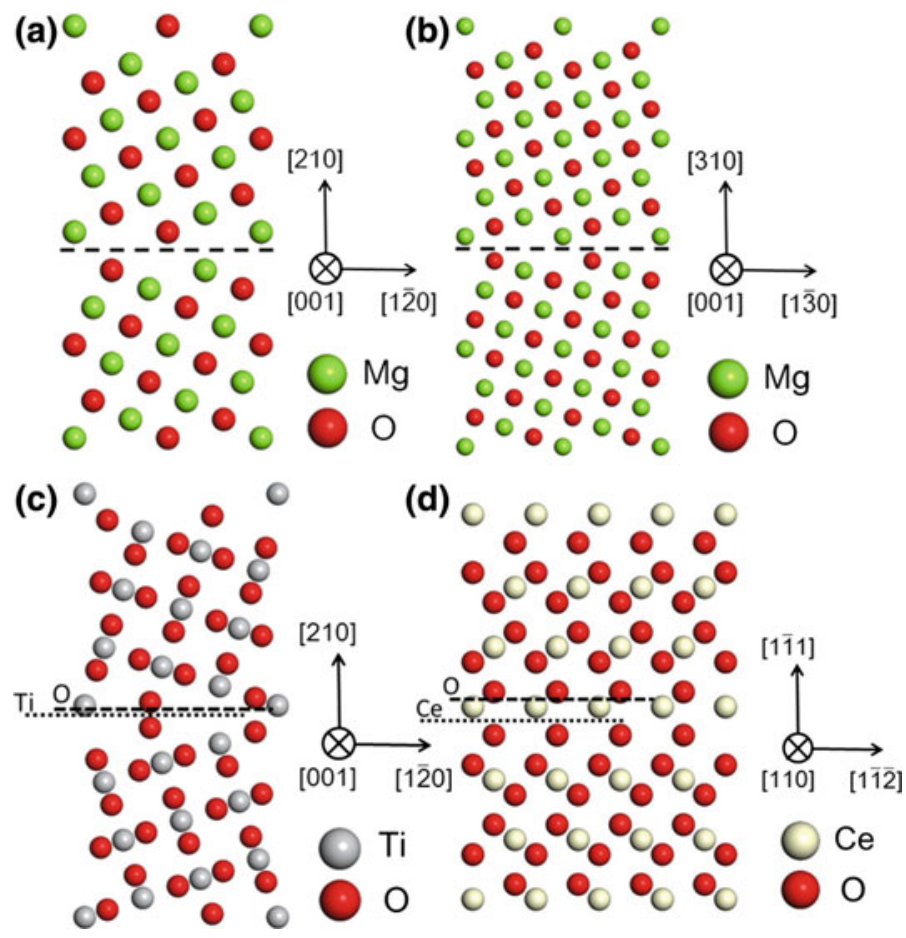

Fig. 8.9 Atomic structure of a $\Sigma 5[001] /(210), \mathbf{b} \Sigma 5[001] /(310) \mathrm{GBs}$ of $\mathrm{MgO}, \mathbf{c} \Sigma 5[001] /(210) \mathrm{GB}$ of $\mathrm{TiO}_{2}$, and $\Sigma 3[110] /(111) \mathrm{GB}$ of $\mathrm{CeO}_{2}[16]$

respectively so that the kernel is not biased to 0 or 1 . The random selection number for the initial calculation was set to 5 , with the actual size of the three-dimensional rigid body translations in each xyz-direction acting as descriptors. Namely, smaller numbers than the above metal cases were used due to the higher computational cost of the oxide simulations.

For structure optimization and energy calculation, static lattice calculations with an empirical potential were performed using a general utility lattice program (GULP) code [21]. Buckingham-type potentials were used for $\mathrm{MgO}$ (Catlow et al. [27]), $\mathrm{TiO}_{2}$ (Bandura et al. [28]), and $\mathrm{CeO}_{2}$ (Minervini et al. [29]).

Regarding the convergence criteria in the Kriging method, the structure searching continues until five structures which have the identical lowest grain boundary energy are found. In this case, the grain boundary energies within $0.005 \mathrm{~J} / \mathrm{m}^{2}$ were judged to be the same. Until these convergence criteria are met, the Kriging algorithm continues searching for the lowest energy configuration.

Figure $8.10 \mathrm{a}$, b show the obtained $\Sigma 5[001](210)$ and $\Sigma 5[001](310)$ grain boundaries of rock-salt- $\mathrm{MgO}$, which has a single grain boundary termination plane as shown in Fig. 8.9a, b. Previously reported structures are overlaid on the structures calculated herein using black or white circles [30,31]. The number of candidate configurations for $\Sigma 5[001](210)$ and $\Sigma 5[001](310)$ structures equals 28,896 and 40,635 respectively. In 
(a)

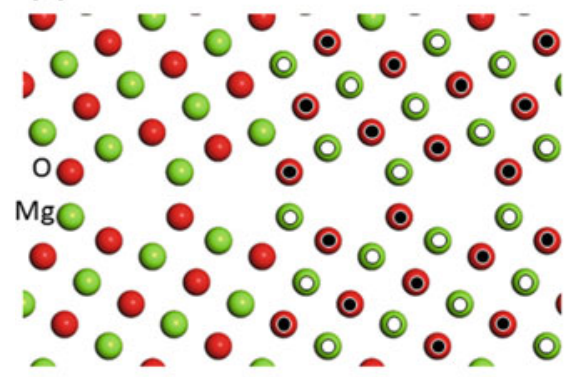

(c)

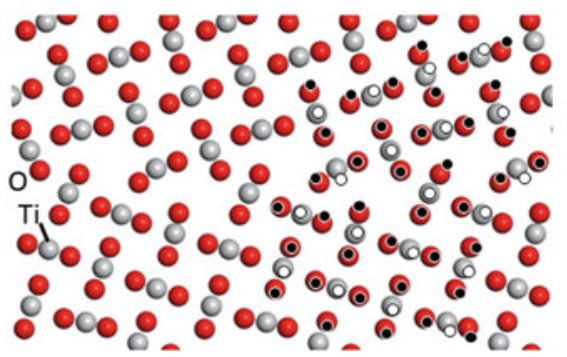

(b)

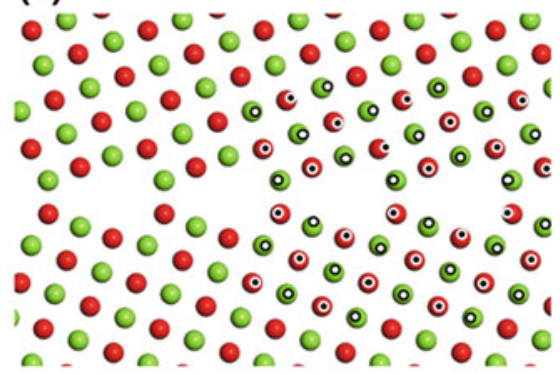

(d)

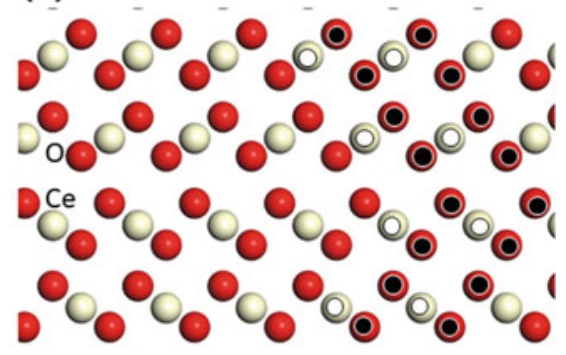

Fig. 8.10 Calculated structures of $\mathrm{MgO}$ a $\Sigma 5(210), \mathbf{b} \Sigma 5(310), \mathbf{c} \mathrm{TiO}_{2} \Sigma 5(210)$, and $\mathbf{d ~} \mathrm{CeO}_{2} \Sigma 3$ (111) GBs [16]

the conventional method, structure optimization and energy calculations for all candidates need to be performed to determine the most stable structure. Conversely, using the Kriging method, we have succeeded in determining the most stable structures for $\Sigma 5[001](210)$ and $\Sigma 5[001](310)$ by performing only 18 and 15 calculations respectively, including initial random calculations.

We performed the Kriging method several times and confirmed that the number of calculations needed to reach convergence in these cases is 14-22, including the initial random sampling. This variation comes from the selection of the initial sampling. However, we would like to emphasize that the Kriging method is clearly powerful to search the most stable structure.

To confirm the applicability of this method to more complex structures, we applied it to $\mathrm{TiO}_{2}$ and $\mathrm{CeO}_{2}$ grain boundaries. The most stable structure of the rutile- $\mathrm{TiO}_{2} \Sigma 5[001] /(210)$ grain boundary is shown in Fig. 8.10c. To maintain charge neutrality, the termination planes were set to $\mathrm{Ti}$ and $\mathrm{O}$ on their respective sides. A total of 21,630 structures and energies need to be computed in order to find the most stable structure using conventional brute force calculations, while the Kriging method can find the most stable structure by performing only 42 calculations, achieving a more than 500-fold efficiency. In addition, the obtained structure was compared with the previously reported one (Fig. 8.10c) [32], clearly showing that the structure determined by the present strategy was correct. 
Next, the present method was applied to the fluorite- $\mathrm{CeO}_{2} \Sigma 3[110](111)$ grain boundary. In contrast to the other three grain boundaries, this one possesses a [110] rotation axis. Notably, the Kriging method can determine the most stable structure (which is in agreement with the one reported previously [33]) using only 12 calculations. These results indicate that the Kriging method is applicable to complex oxides and can potentially achieve efficiency improvements by factors of $\sim 10^{3}-10^{4}$ over the conventional all-candidate calculation method.

Finally, the reasons behind the broad applicability of the Kriging method are discussed. As mentioned above, the Kriging method searches for stable structures in a three-dimensional data set, as shown in Fig. 8.5b, with extrapolation of this data

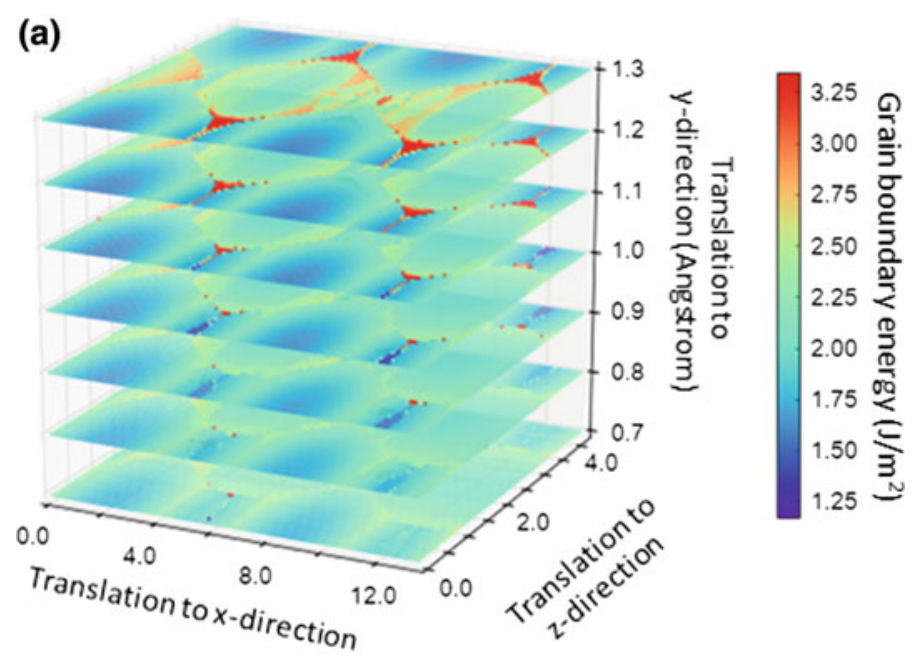

(b)

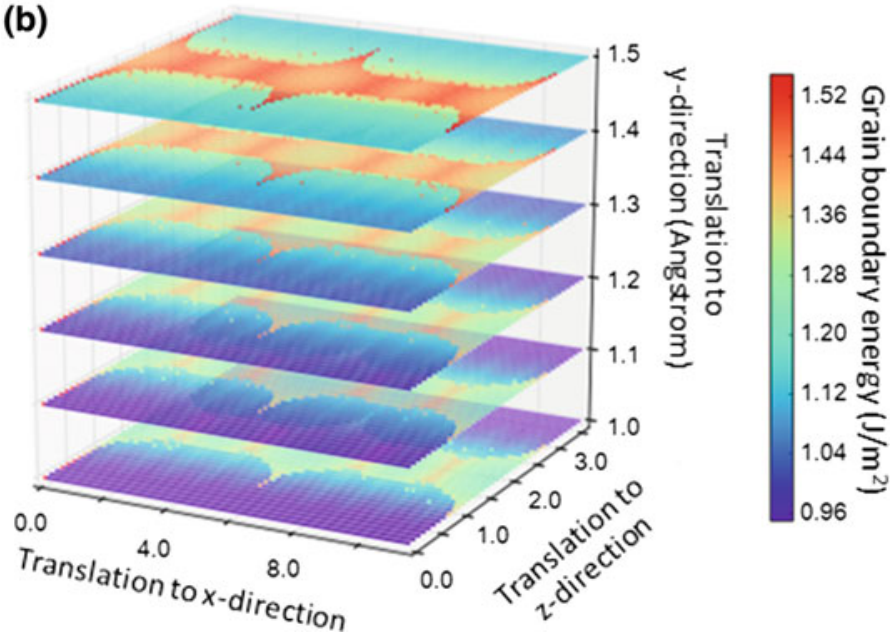

Fig. 8.11 Data space for structure searching. a Grain boundary energy plots in the data space for $\Sigma 5[001](310) \mathrm{GB}$ of $\mathrm{MgO}$ and $\mathbf{b} \Sigma 5[001](310) \mathrm{GB}$ of $\mathrm{Cu}[15,16]$ 
space performed using the Gaussian process. The success of the Kriging method indicates the suitability of this extrapolation method for the present data space, with data spaces for $\mathrm{MgO} \Sigma 5[001](310)$ and $\mathrm{Cu} \Sigma 5$ [001](310) compared in Fig. 8.11a, b. The corresponding $\mathrm{Cu}$ data space was obtained in previous studies [12, 15]. Although these data spaces appear to be different from each other, their energy profiles are similar. Namely, the grain boundary energy gradually and continuously changes with changing rigid body translation, with no discrete large energy changes present in the data space. This fact indicates that the Kriging method is applicable for grain boundaries possessing a continuous energy surface.

\subsection{Microscopic Approach for Interfaces}

\subsubsection{Scanning Transmission Electron Microscopy (STEM)}

STEM is one branch of TEM techniques that has been extensively used for characterizing interface structures in many materials and devices. In recent years, STEM combined with aberration correction technology has enabled direct atom-by-atom imaging via annular dark-field (ADF) imaging. ADF imaging uses a doughnut-shaped annular detector to selectively collect high-angle scattered electrons, building up images from the variation in this signal with probe position in a raster scan. Since the integrated intensity of high-angle scattered electrons strongly scales with the atomic number of the atoms under the probe, this imaging (so-called Z-contrast imaging) can sensitively visualize heavy element atoms [34]. However, $\mathrm{ADF}$ can seldom reliably visualize light elements due to their weak power to scatter electrons at higher angles. While ADF imaging mainly uses electrons scattered at high-angles to form atom images, there are many other possible detector geometries for collecting electron signals and forming images. One is known as annular bright-field $(\mathrm{ABF})$ imaging, which involves the selective collection of electrons inside the bright-field disk via a small annular detector [35]. It has been shown that ABF imaging can directly visualize light atoms and can even directly visualize $\mathrm{H}$ atom columns inside compound materials [36, 37]. Since ADF and ABF images can be obtained simultaneously from the same sample positions, both heavy and light element atomic structures can now be directly visualized by combining these two images. However, it is still very difficult to identify the atomic species using only the ADF and ABF image contrasts, especially at interface regions where structure and chemistry are drastically changing. Since STEM uses an atomic-scale electron probe, STEM-based analytical techniques such as energy-dispersive X-ray spectroscopy (EDS) and electron energy loss spectroscopy (EELS) can also achieve atomic resolution. In particular, by utilizing ultrasensitive silicon drift detectors (SDDs) with much higher count rates, atomic-scale EDS mapping is now becoming possible [38]. This capability should be very powerful to directly characterize dopant/impurity segregation behaviors in grain boundaries and heterointerfaces. 
Thus, atomic-resolution STEM combined with spectroscopy will become an indispensable technique for characterizing atomic-scale structures and chemistry of interfaces.

\subsubsection{Interface Structures Using Aberration-Corrected STEM}

Two interface characterization studies using aberration-corrected STEM and EDS are highlighted in this section. One is on the solute segregation in a GB of ceramics [39] and the other is on the impurity segregation in a metal/ceramic heterointerface [40]. These studies demonstrate that atomic-resolution STEM is a powerful tool for directly understanding very complex segregation phenomena in materials.

\subsubsection{Solute Segregation Behavior of a $\sum 3$ Grain Boundary in Yttria Stabilized Zirconia [39]}

$\mathrm{ZrO}_{2}$ doped with $\mathrm{Y}_{2} \mathrm{O}_{3}$ (YSZ) is one of the most important materials for use as an electrolyte in solid oxide fuel cells, where the overall ionic conductivity is strongly affected by the presence of GBs; such an effect may potentially originate from GB chemical inhomogeneity. Previous studies have shown that the $\mathrm{Y}$ solute atoms segregate to the GBs, and the amount of segregation is strongly dependent on the GB characteristics [41]. However, the atomic-scale mechanism of how Y solute actually segregate to GBs is still not well understood. In this study, we show an atomic-scale EDS mapping of a $\Sigma 3[110] /\{111\}$ model GB in YSZ.

Figure 8.12 shows an ADF STEM image of the $\Sigma 3[110] /\{111\}$ grain boundary of YSZ, where the GB atomic arrangement is in good agreement with previous high-resolution TEM studies [41]. However, it is almost impossible to distinguish $\mathrm{Zr}$ and $\mathrm{Y}$ atoms from the STEM image alone. Then, atomic-resolution STEM-EDS mapping was carried out in order to distinguish the two atomic components. Figure 8.13a, b show atomic-resolution EDS maps around the GB, where the $\mathrm{Y}$ and $\mathrm{Zr}$ maps clearly reveal the formation of characteristic ordered segregation structures. The intensity variation is further highlighted in the corresponding intensity profile shown in Fig. 8.13c, d.

It is noteworthy that in some atomic column layers, $\mathrm{Y}$ atoms are obviously depleted. This indicates that the $\mathrm{Y}$ atoms are not simply substituting in all of the cation sites around the GB to form segregation structures. Thus, we experimentally found that $\mathrm{Y}$ solute segregation formed atomically ordered extended structures across the GB within a range of approximately $3 \mathrm{~nm}$. These experimental results are in good agreement with large-scale Monte Carlo simulations [39]. The simulation suggested that such processes can be driven by both the site-dependent segregation of $\mathrm{Y}$ due to strain and $\mathrm{Y}-V_{O}$ interactions. Thus, recent advanced microscopy 


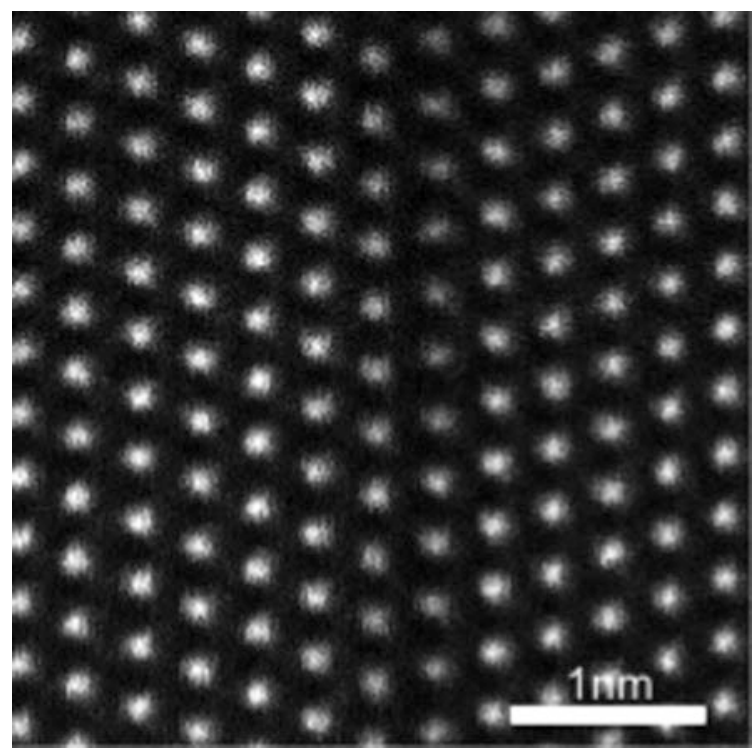

Fig. 8.12 ADF STEM image of the $\Sigma 3[110] /\{111\}$ grain boundary of YSZ (adopted from Ref: [39])
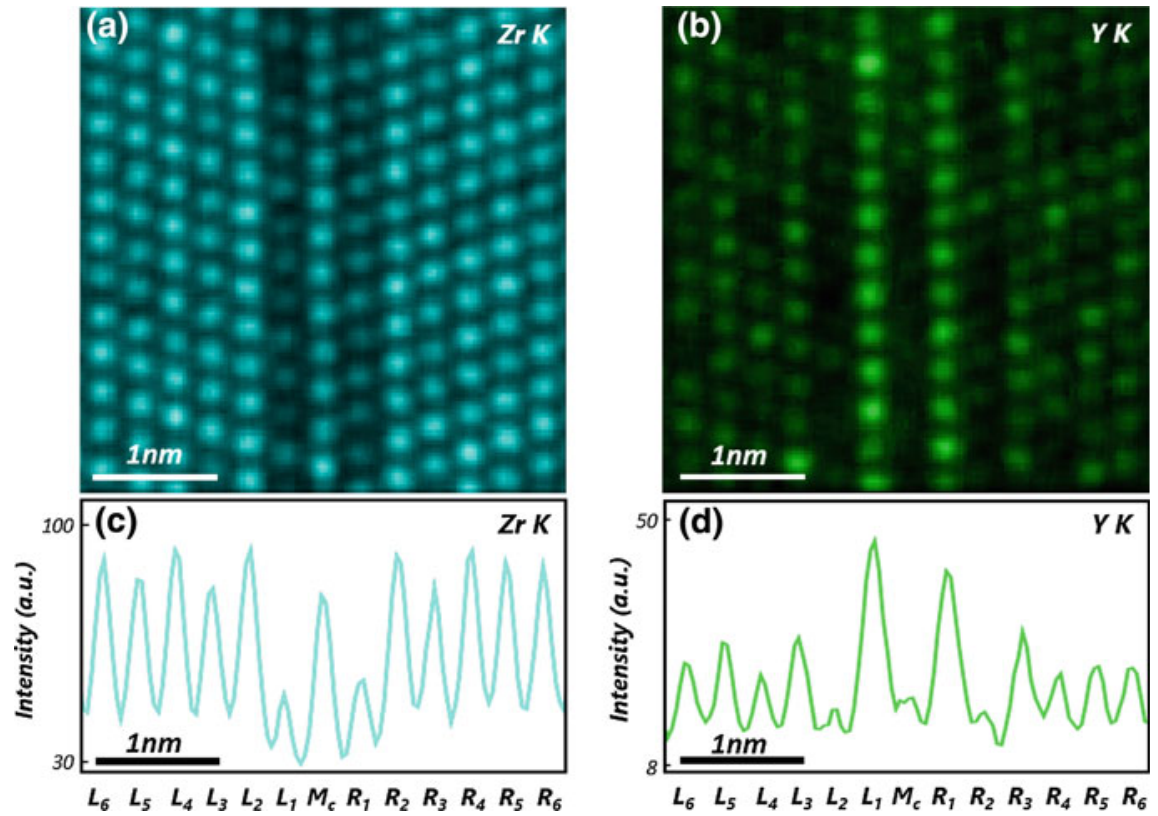

Fig. 8.13 a, b, EDS elemental maps for a Zr K map and b Y K map. c, d, normalized intensity profiles derived by summing the X-ray counts in the maps in the direction parallel to the GB for c Zr K and d Y K (adopted from Ref: [39]) 
combined with theory can shed new light on the fundamental mechanism of solute segregation behaviors in GBs.

\subsubsection{Dopant Segregation Behavior in a Metal/Ceramic Interface [40]}

Heterostructures between metals and ceramics have been widely used for power electronic devices requiring both high thermal performance and reliability in harsh environments. Since interfaces play a critical role in many properties, a fundamental understanding of the interface structure and formation mechanism is vitally important. One important possibility for obtaining heterointerfaces with better properties is to control dopant/impurity segregation behaviors. However, it has been very challenging to directly observe segregation structures at atomic dimensions in heterointerfaces. Here, atomic-resolution STEM-EDS mapping is shown to be a powerful tool for directly determining segregation structures in metal/ceramic heterointerfaces.

Figure 8.14 shows simultaneous cross-sectional (a) ADF and (b) ABF STEM images of an $\mathrm{Al}$ alloy (containing $\mathrm{Si}$ and $\mathrm{Mg}$ as major dopants) /AlN interface formed using a liquid phase bonding technique [40]. In the AlN bulk region, compared to the ADF image, the columns with weaker intensities in the ABF image correspond to the $\mathrm{N}$ columns, and the interface of AlN should be Al-polar. The atomic structures of the Al alloy region were not clearly resolved because the present viewing direction is not well-aligned along the certain high symmetry crystallographic axis. To clearly identify the interface atomic structure, we show noise-filtered images of the interface core region in Fig. 8.14c, d. From these images, we can divide the interface core region into three different layered structures labeled as the 1st, 2nd, and 3rd layers. Considering both ADF and ABF STEM images, the 1st, 2nd and 3rd layer interface structures are anion-cation-anion, cation-anion, and anion layers, respectively. However, it is difficult to determine the detailed atomic structure of the three layers from the STEM image contrast since dopant elements such as $\mathrm{Al}$ and $\mathrm{Mg}$ with close atomic numbers may coexist. Thus, we performed atomic-resolution chemical mapping using STEM-EDS.

Figure 8.15 shows atomically resolved chemical maps of the Al alloy/AlN interface using STEM-EDS. The elemental maps of $\mathrm{Al}, \mathrm{N}, \mathrm{O}, \mathrm{Mg}$, and $\mathrm{Si}$ are shown in comparison with an ABF STEM image and structure model [40]. We found that the highest signal in $\mathrm{Mg}$ and $\mathrm{O}$ maps is located at the 1st interfacial layer, but that of $\mathrm{Si}$ is slightly shifted within the $\mathrm{Al}$ alloy region. This indicates that these dopant elements should occupy different atomic layers at the interface region. In the 1st layer, $\mathrm{Mg}$ atoms are concentrated to a single atomic column layer, whereas $\mathrm{O}$ atoms are concentrated to the top and bottom of the $\mathrm{Mg}$ layer. Considering the bonding distances and angles between $\mathrm{Mg}$ and $\mathrm{O}$ columns in the 1st layer, this structure is very similar to a $\mathrm{MgO}_{6}$ octahedron with rock salt structure. In the 2nd layer, a local maximum of $\mathrm{Al}$ can be found at the cation columns. Thus, the cation columns can be identified as $\mathrm{Al}$ columns. $\mathrm{O}$ and $\mathrm{N}$ could not be separated in the 2 nd layer, 

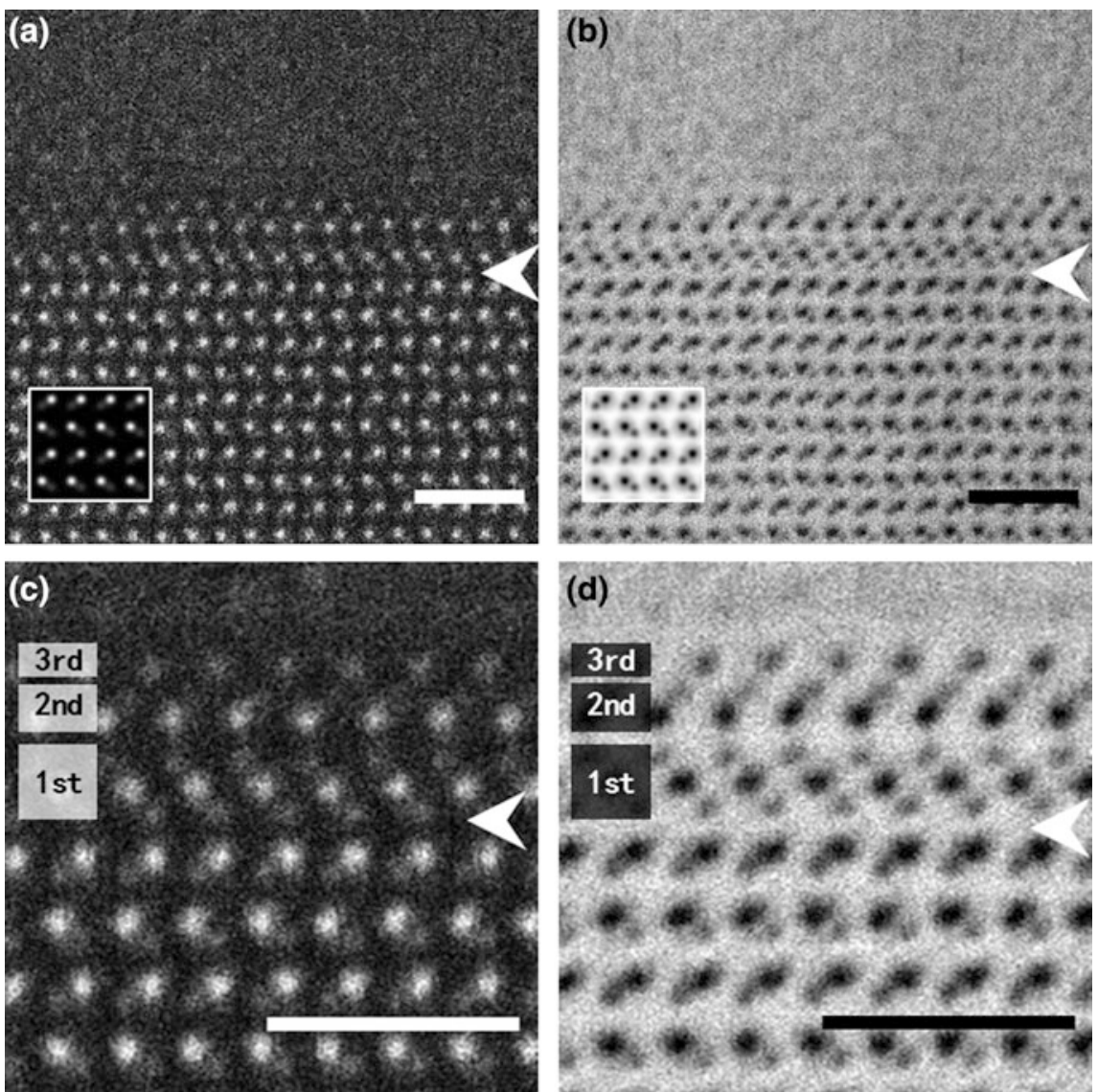

Fig. 8.14 Simultaneously obtained atomic-resolution ADF $\mathbf{a}$ and ABF $\mathbf{b}$ STEM images of an Al alloy/AlN interface (adopted from Ref: [40]). Calculated images of AlN bulk are superimposed in the lower left in $\mathbf{a}$ and $\mathbf{b}$. The magnified views are shown in $\mathbf{c}$ and $\mathbf{d}$

but the ABF image contrast of the 2nd layer is similar to the Al-N contrast in the AlN bulk structure, although with inverted polarity (N-polar). Thus, we consider that the main structure of the 2 nd layer is an $\mathrm{AlN}_{4}$ tetrahedral monolayer. The polarity of this layer is inverted from the AlN substrate due to the presence of the $\mathrm{MgO}$ interlayer. Theoretical simulations suggested that the interface between $\mathrm{Al}$ metal and $\mathrm{N}$-polar AlN is much more energetically stable than that between $\mathrm{Al}$ metal and Al-polar AlN.

Thus, Al alloy /AlN heterointerfaces should be stabilized by the formation of self-organized atomic-scale layered structures with $\mathrm{Mg}$ dopant segregation. Atomic-resolution STEM-EDS is a powerful tool for directly determining heterointerface structures with dopant/impurity segregation. 


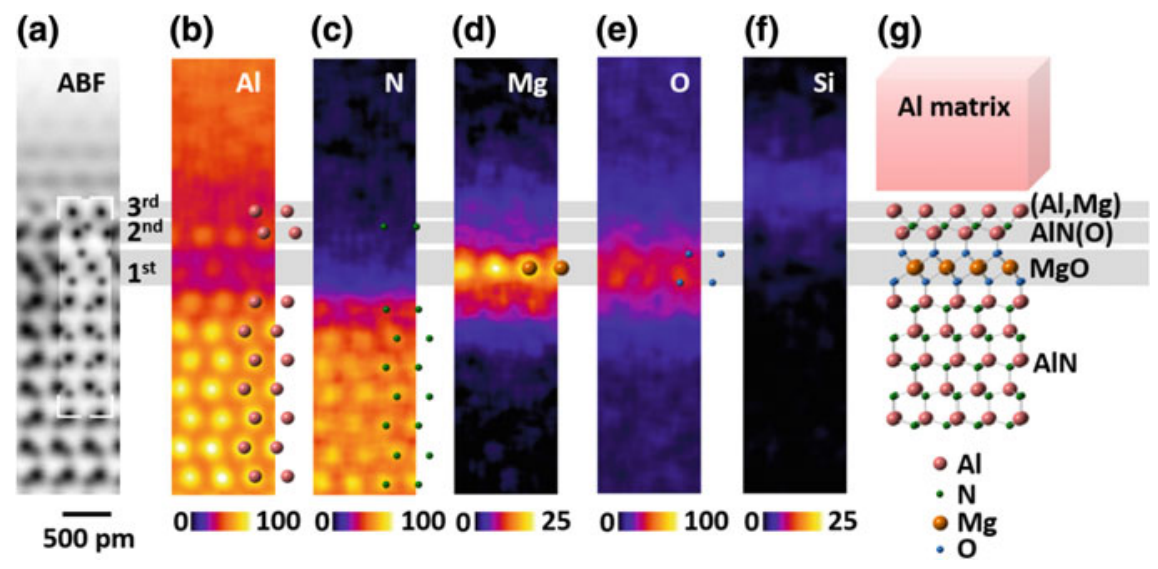

Fig. 8.15 a The averaged ABF STEM image and the corresponding elemental maps of $\mathbf{b} \mathrm{Al}, \mathbf{c} \mathrm{N}$, d $\mathrm{Mg}$, e $\mathrm{O}$, and f $\mathrm{Si}$, respectively (adopted from Ref: [40]). The structure model of the heterointerface $\mathbf{g}$ determined from the experimental results is shown

Acknowledgements This work was supported by a Grant-in-Aid for Scientific Research on Innovative Areas "Nano Informatics" (Grant No. JP25106003) from the Japan Society for the Promotion of Science (JSPS).

\section{References}

1. Y. Ikuhara, Grain boundary atomic structures and light-element visualization in ceramics: combination of Cs-corrected scanning transmission electron microscopy and first-principles calculations. J. Electron. Microsc. (Tokyo) 60, 173-188 (2011). https://doi.org/10.1093/ jmicro/dfr049

2. A. Sutton, R. Balluffi, Interfaces in Crystalline Materials (Clarendon, 1995)

3. J.P. Buban, K. Matsunaga, J. Chen, N. Shibata, W.Y. Ching, T. Yamamoto, Y. Ikuhara, Grain boundary strengthening in alumina by rare earth impurities. Science 311(2006) 212-215. https://doi.org/10.1126/science.1119839

4. K. Matsunaga, H. Nishimura, H. Muto, T. Yamamoto, Y. Ikuhara, Direct measurements of grain boundary sliding in yttrium-doped alumina bicrystals. Appl. Phys. Lett. 82, 1179-1181 (2003). https://doi.org/10.1063/1.1555690

5. T. Mizoguchi, H. Ohta, H.-S. Lee, N. Takahashi, Y. Ikuhara, Controlling interface intermixing and properties of SrTiO3-based superlattices. Adv. Funct. Mater. 21, 22582263 (2011). https://doi.org/10.1002/adfm.201100230

6. H. Ohta, S. Kim, Y. Mune, T. Mizoguchi, K. Nomura, S. Ohta, T. Nomura, Y. Nakanishi, Y. Ikuhara, M. Hirano, H. Hosono, K. Koumoto, Giant thermoelectric Seebeck coefficient of a two-dimensional electron gas in SrTiO3. Nat. Mater. 6, 129-134 (2007). https://doi.org/10. 1038/nmat1821

7. H. Sawada, N. Shimura, F. Hosokawa, N. Shibata, Y. Ikuhara, Resolving 45-pm-separated Si-Si atomic columns with an aberration-corrected STEM. Microscopy 64, 213-217 (2015). https://doi.org/10.1093/jmicro/dfv014

8. S.J. Pennycook, P.D. Nellist, Scanning Transmission Electron Microscopy (Springer New York, New York, 2011). https://doi.org/10.1007/978-1-4419-7200-2

9. N. Tanaka, Scanning transmission electron microscopy of nanomaterials, in ed. by N. Tanaka (Imperial College Press, London, 2014), pp. i-xliv. https://doi.org/10.1142/9781848167902_ fmatter 
10. S. Ranganathan, On the geometry of coincidence-site lattices. Acta Crystallogr. 21, 197-199 (1966). https://doi.org/10.1107/S0365110X66002615

11. D. Brandon, The structure of high-angle grain boundaries. Acta Metall. 14, 1479-1484 (1966). https://doi.org/10.1016/0001-6160(66)90168-4

12. S. Kiyohara, H. Oda, T. Miyata, T. Mizoguchi, Prediction of interface structures and energies via virtual screening. Sci. Adv. 2, e1600746-e1600746 (2016). https://doi.org/10.1126/ sciadv. 1600746

13. A.L.-S. Chua, N.A. Benedek, L. Chen, M.W. Finnis, A.P. Sutton, A genetic algorithm for predicting the structures of interfaces in multicomponent systems. Nat. Mater. 9, 418-422 (2010). https://doi.org/10.1038/nmat2712

14. G. Schusteritsch, C.J. Pickard, Predicting interface structures: from SrTiO3 to graphene. Phys. Rev. B. 90, 35424 (2014). https://doi.org/10.1103/PhysRevB.90.035424

15. S. Kiyohara, H. Oda, K. Tsuda, T. Mizoguchi, Acceleration of stable interface structure searching using a kriging approach. Jpn. J. Appl. Phys. 55, 2-6 (2016). https://doi.org/10. 7567/JJAP.55.045502

16. S. Kikuchi, H. Oda, S. Kiyohara, T. Mizoguchi, Bayesian optimization for efficient determination of metal oxide grain boundary structures. Phys. B Condens. Matter. (2017) (in press). https://doi.org/10.1016/j.physb.2017.03.006

17. T. Ueno, T.D. Rhone, Z. Hou, T. Mizoguchi, K. Tsuda, COMBO: an efficient Bayesian optimization library for materials science, Mater. Discov. 10-13, (2016). https://doi.org/10. 1016/j.md.2016.04.001

18. R. Gómez-Bombarelli, J. Aguilera-Iparraguirre, T.D. Hirzel, D. Duvenaud, D. Maclaurin, M. A. Blood-Forsythe, H.S. Chae, M. Einzinger, D.-G. Ha, T. Wu, G. Markopoulos, S. Jeon, H. Kang, H. Miyazaki, M. Numata, S. Kim, W. Huang, S.I. Hong, M. Baldo, R.P. Adams, A. Aspuru-Guzik, Design of efficient molecular organic light-emitting diodes by a high-throughput virtual screening and experimental approach. Nat. Mater. 15, 1120-1127 (2016). https://doi.org/10.1038/nmat4717

19. D. Wolf, Structure-energy correlation for grain boundaries in F.C.C. metals-III. Symmetrical tilt boundaries. Acta Metall. Mater. 38, 781-790 (1990). https://doi.org/10.1016/0956-7151 (90)90030-K

20. G. Hasson, J.-Y. Boos, I. Herbeuval, M. Biscondi, C. Goux, Theoretical and experimental determinations of grain boundary structures and energies: correlation with various experimental results. Surf. Sci. 31, 115-137 (1972). https://doi.org/10.1016/0039-6028(72)90256-7

21. J.D. Gale, GULP: a computer program for the symmetry-adapted simulation of solids. J. Chem. Soc. Faraday Trans. 93, 629-637 (1997)

22. F. Cleri, V. Rosato, Tight-binding potentials for transition metals and alloys. Comput. Simul. Mater. Sci. 205, 233-253 (1991). https://doi.org/10.1007/978-94-011-3546-7_11

23. P. Ballo, V. Slugeň, Grain boundary sliding and migration in copper: the effect of vacancies. Comput. Mater. Sci. 33, 491-498 (2005). https://doi.org/10.1016/j.commatsci.2004.09.049

24. M.A. Tschopp, D.L. Mcdowell, Asymmetric tilt grain boundary structure and energy in copper and aluminium. Philos. Mag. 87, 3871-3892 (2007). https://doi.org/10.1080/ 14786430701455321

25. H. Nakashima, M. Takeuchi, Grain boundary energy and structure of $\alpha-\mathrm{Fe}<110>$ symmetric tilt boundary, Tetsu-to-Hagane. 86(2000) 357-362. https://doi.org/10.2355/tetsutohagane1955. 86.5_357

26. R. Kurtz, H. Heinisch, The effects of grain boundary structure on binding of He in Fe. J. Nucl. Mater. 329-333, 1199-1203 (2004). https://doi.org/10.1016/j.jnucmat.2004.04.262

27. A. Catlow, Chapter IO Interionic Potentials in Ionic Solids, (n.d.)

28. A.V. Bandura, J.D. Kubicki, Derivation of force field parameters for TiO2-H2O systems from ab initio calculations (2003). https://doi.org/10.1021/JP034093T

29. L. Minervini, M.O. Zacate, R.W. Grimes, Defect cluster formation in M2O3-doped CeO2. Solid State Ionics 116, 339-349 (1999). https://doi.org/10.1016/S0167-2738(98)00359-2 
30. B.P. Uberuaga, X.-M. Bai, P.P. Dholabhai, N. Moore, D.M. Duffy, Point defect-grain boundary interactions in $\mathrm{MgO}$ : an atomistic study. J. Phys. Condens. Matter 25, 355001 (2013). https://doi.org/10.1088/0953-8984/25/35/355001

31. Y. Yan, M.F. Chisholm, G. Duscher, A. Maiti, S.J. Pennycook, S.T. Pantelides, Impurity-induced structural transformation of a $\mathrm{MgO}$ grain boundary, Phys. Rev. Lett. 81 (1998), 3675-3678. https://doi.org/10.1103/PhysRevLett.81.3675

32. S.B. Sinnott, R.F. Wood, S.J. Pennycook, Ab initio calculations of rigid-body displacements at the $\Sigma 5$ (210) tilt grain boundary in TiO2. Phys. Rev. B. 61, 15645-15648 (2000). https:// doi.org/10.1103/PhysRevB.61.15645

33. B. Feng, H. Hojo, T. Mizoguchi, H. Ohta, S.D. Findlay, Y. Sato, N. Shibata, T. Yamamoto, Y. Ikuhara, Atomic structure of a $\Sigma 3[110] /(111)$ grain boundary in $\mathrm{CeO} 2$. Appl. Phys. Lett. 100, 73109 (2012). https://doi.org/10.1063/1.3682310

34. S.J. Pennycook, L.A. Boatner, Chemically sensitive structure-imaging with a scanning transmission electron microscope. Nature 336, 565-567 (1988). https://doi.org/10.1038/ $336565 \mathrm{a} 0$

35. S.D. Findlay, N. Shibata, H. Sawada, E. Okunishi, Y. Kondo, T. Yamamoto, Y. Ikuhara, Robust atomic resolution imaging of light elements using scanning transmission electron microscopy. Appl. Phys. Lett. 95, 2-4 (2009). https://doi.org/10.1063/1.3265946

36. S.D. Findlay, T. Saito, N. Shibata, Y. Sato, J. Matsuda, K. Asano, E. Akiba, T. Hirayama, Y. Ikuhara, Direct imaging of hydrogen within a crystalline environment. Appl. Phys. Express 3, 6-9 (2010). https://doi.org/10.1143/APEX.3.116603

37. R. Ishikawa, E. Okunishi, H. Sawada, Y. Kondo, F. Hosokawa, E. Abe, Direct imaging of hydrogen-atom columns in a crystal by annular bright-field electron microscopy. Nat. Mater. 10, 278-281 (2011). https://doi.org/10.1038/nmat2957

38. M.W. Chu, S.C. Liou, C.P. Chang, F.S. Choa, C.H. Chen, Emergent chemical mapping at atomic-column resolution by energy-dispersive X-ray spectroscopy in an aberration-corrected electron microscope, Phys. Rev. Lett. 104(2010), 1-4. https://doi.org/10.1103/PhysRevLett. 104.196101

39. B. Feng, T. Yokoi, A. Kumamoto, M. Yoshiya, Y. Ikuhara, N. Shibata, Atomically ordered solute segregation behaviour in an oxide grain boundary. Nat. Commun. 7, 11079 (2016). https://doi.org/10.1038/ncomms11079

40. A. Kumamoto, N. Shibata, K. Nayuki, T. Tohei, N. Terasaki, Y. Nagatomo, T. Nagase, K. Akiyama, Y. Kuromitsu, Y. Ikuhara, Atomic structures of a liquid-phase bonded metal/nitride heterointerface. Sci. Rep. 6, 22936 (2016). https://doi.org/10.1038/srep22936

41. N. Shibata, F. Oba, T. Yamamoto, Y. Ikuhara §, Structure, energy and solute segregation behaviour of [110] symmetric tilt grain boundaries in yttria-stabilized cubic zirconia. Philos. Mag. 84, 2381-2415 (2004). https://doi.org/10.1080/14786430410001693463

Open Access This chapter is licensed under the terms of the Creative Commons Attribution 4.0 International License (http://creativecommons.org/licenses/by/4.0/), which permits use, sharing, adaptation, distribution and reproduction in any medium or format, as long as you give appropriate credit to the original author(s) and the source, provide a link to the Creative Commons license and indicate if changes were made.

The images or other third party material in this chapter are included in the chapter's Creative Commons license, unless indicated otherwise in a credit line to the material. If material is not included in the chapter's Creative Commons license and your intended use is not permitted by statutory regulation or exceeds the permitted use, you will need to obtain permission directly from the copyright holder. 